Lymphology 53 (2020) 55-62

\title{
A HISTORICAL ACCOUNT OF THE ROLE OF EXERCISE IN THE PREVENTION AND TREATMENT OF CANCER-RELATED LYMPHEDEMA
}

\author{
K. Johansson, S. Hayes
}

Department of Health Sciences (KJ), Lund University, Lund, Sweden, and Menzies Health Institute Queensland (SH), Griffith University, Queensland, Australia

\section{ABSTRACT}

In the absence of guidance from scientific evidence, a range of lymphedema prevention and management, guidelines were developed by relevant organizations around the world. These became publicly available, promoted and endorsed, particularly to women with breast cancer. The recommendations advised avoidance of any activity that could overload or restrict the lymphatic system and need for caution when participating in specific physical activities. However, over the past 20 years evidence has accumulated which has significantly challenged the safety of these recommendations, in particular for those with cancer. There now exists consistent and compelling evidence in support of exercise following a diagnosis of cancer. Participating in exercise during and following cancer treatment improves function and quality of life, reduces treatment-related morbidity, and may improve survival. Further, exercise, including resistance exercise at moderate or high load, is considered safe for those at risk-or with lymphedema. That is, exercise has not been shown to cause or worsen cancer-related lymphedema. This article provides a historical account of the advice given to patients in the prevention and management of lymphedema and how this advice has evolved.
Keywords: lymphedema, physical activity, exercise, cancer, prevention, treatment

Lymphedema manifests as a consequence of lymphatic system insufficiency where lymph transport capacity is lower than lymph load (1). It is a debilitating condition of worldwide concern and is prevalent in developing and developed countries (2). The major contributing factors to its development differ between countries. In developed nations, it is largely associated with the presence of chronic diseases, including obesity, type II diabetes, and cardiovascular disease, although is most well-known as a consequence of treatment for cancer (2).

Removal of lymph nodes and receipt of adjuvant therapy, such as radiotherapy and chemotherapy have been identified as the primary treatment-related risk factors for the development of cancer-related lymphedema (3). These represent common treatment options for the most prevalent cancers, including breast, prostate, colorectal, and melanoma, but also for the treatment of other solid cancers, such as gynecological, genitourinary, and head and neck cancers. Following a meta-analysis, the incidence of upper-limb lymphedema associated with breast cancer has been estimated to be $21 \%$ (3), however with more invasive treatment the 
incidence is higher (4). While less is known about the incidence of lymphedema associated with other cancers, lower-limb lymphedema associated with gynecological and prostate cancer is considered to be at least as common as breast cancer-related lymphedema, and potentially more debilitating (5).

The presence of lymphedema has a cascade of adverse physical, functional, psychosocial, emotional, and financial ramifications (6). Further, treatment options are considered time consuming, costly and patient- and clinician-intensive with success variable on the stage and duration of lymphedema (7). These factors have contributed to lymphedema being labeled the most feared cancer treatment-related adverse effect (8). This fear, not surprisingly, lead to clinicians and patients asking 'how can risk of lymphedema be reduced and what can be done to avoid exacerbations for those with lymphedema?'. In the absence of guidance from scientific evidence, recommendations made by clinicians to their patients were empirically based and advised avoidance of activities that may contribute to an increase in lymph load or restrict lymph flow. As such, in the 170 s through to the late 190 s, those at riskor with lymphedema were recommended "remedial" exercise only (Fig. 1), that is, specific range of motion exercises of the limb, done slowly and without weights. These were the kind of exercises that may have been advised to all patients (irrespective of the perceived risk of lymphedema) as part of standard rehabilitation advice following cancer treatment (in particular, surgical and radiation treatment). It was believed that this type of movement therapy could aid functional recovery from treatment, while also supporting venous and lymphatic flow through muscle contractions without increasing blood flow and therefore lymphatic load (9). In contrast, participation in vigorous or repetitive exercise was considered contraindicated. It was hypothesized that the increase in lymph production created by any exercise that was higher in load or repetition than remedial exercise would outweigh any benefit to lymph transport capacity generated by the muscle contraction. Supporting the theoretical basis of these recommendations were anecdotal accounts by patients to doctors regarding events (e.g., the lifting of a heavy weight, such as a grandchild; painting a wall) that precipitated the development of their lymphedema. As such, the common 'rest is best' advice previously widely given to patients following a diagnosis of cancer was considered even more relevant for those at risk of developing lymphedema or with cancer-related lymphedema.

Over time, a range of lymphedema prevention and management guidelines were developed and became publicly available, promoted and endorsed, particularly to women with breast cancer (Fig. 1). The recommendations advised avoidance of any activity that could 'overload' or 'restrict' the lymphatic system and need for caution when participating in specific activities. This included a long list of 'do not...' (e.g., do not carry anything heavier than 2 liters of milk, do not engage in repetitive movements of the at-risk limb, do not allow blood pressure measurements to be taken on the side of the at-risk or affected limb) and avoidance or caution in participating in activities, such as washing dishes in hot water (as heat may increase blood flow and lymphatic load), gardening (since an insect bite would increase lymphatic load or a cut would increase risk of infection), and caution with travel by airplane (with need for garment wear if flying). These recommendations were risk-averse, and based on theory rather than evidence and the premise of 'do no harm'. Unfortunately, it is plausible that these guidelines exacerbated, rather than reduced, fear and stigma associated with lymphedema, and concurrently, encouraged reduced and restricted use of at-risk limbs, as well as overall reductions in participation in all types of physical activity (10).

Over the past 20 years, evidence has accumulated which has significantly challenged the safety of these recommen- 


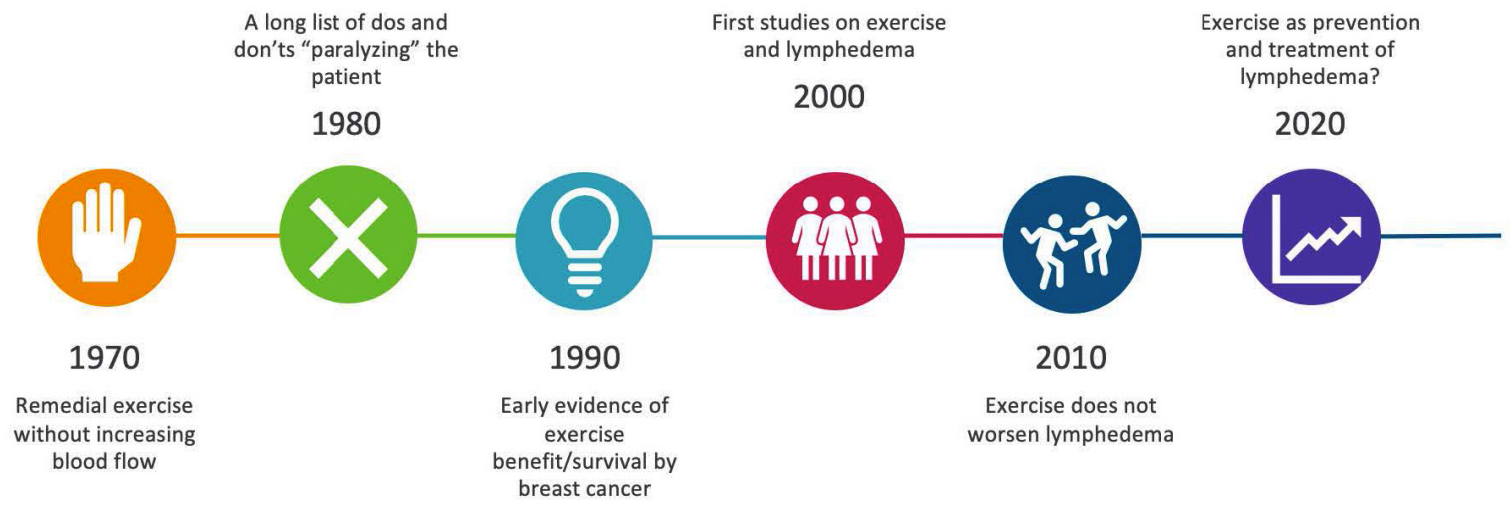

Fig. 1. The evolution of exercise advice for patients with cancer-related lymphedema.

dations, in particular for those with cancer.

More pointedly, it could be argued that scientific findings suggest that instead of protecting those from developing or worsening lymphedema, this advice may have unnecessarily exacerbated one's risk. Much of this argument is based on what we've learned about the relationship between physical activity (including exercise intervention) and survival following cancer. Epidemiological data from 130+ studies involving more than $\mathbf{1 0}$ cancer types has enabled the conduct of metaanalyses to evaluate the relationship between post-cancer physical activity levels and allcause and cancer-specific survival (11). Findings showed that participating in the highest versus lowest levels of post-diagnosis physical activity was significantly associated with reduced all-cause and cancer-specific survival (HR: 0.61, 95\% CI [0.51, 0.73]; and $0.63[0.53,0.75]$, respectively) for all cancers combined, as well as cancer types including breast, prostate, and gynecological cancer, which are known to be associated with lymphedema. Concurrently, findings from cohort studies also suggested that lower physical activity levels were associated with lower musculoskeletal strength and cardiovascular fitness, and higher body weights, and that lower strength and fitness, and higher body mass index, were associated with poorer survival outcomes (although dependent on cancer type) (12-15). It has also been shown that lower levels of physical activity and higher body mass index are associated with increased risk of lymphedema (3). Together, these findings rightly raise doubt on the safety of traditional lymphedema risk reduction and management guidelines.

Over the past twenty years, there has also been an exponential increase in the number of exercise intervention trials conducted involving people with cancer. This extensive body of evidence has been recently reviewed by various steering groups around the world to develop exercise prescription guidelines for cancer patients (16-18). These efforts have led to the endorsement of exercise as being safe for those at risk or with lymphedema. Of particular relevance to the history of exercise in the context of lymphedema is the work published in the late '90s/early 2000s. Several studies reported on arm morbidity (including reduced shoulder range of motion and muscle strength) following breast cancer (19-21), as well as reduced cardiovascular capacity (22). To counteract these adverse physiological changes, participation in Dragon boating was proposed. Dragon boating requires strenuous, repetitive upper-body activity, which was in direct contrast to the recommendations being made at the time to prevent lymphedema. As such, findings published by pioneers in this field (including McKenzie, Harris and Niesen- 
Vertommen), which showed a two-month muscle strength training program followed by an eight-month Dragon Boat competition season did not increase the risk of lymphedema in a group of breast cancer survivors, received international attention (23). Two years later, Box and colleagues published findings from a randomized, controlled trial that evaluated a 6month lymphedema education and progressive exercise program (involving aerobic- and resistance-exercise) for women newly diagnosed with breast cancer $(n=65)$. Results from this trial showed reduced lymphedema incidence in the exercise group compared with the control group (11\% vs $30 \%$ incidence at 2 years post-diagnosis, respectively) (24). Together, these findings set the scene for the exercise intervention studies involving those at risk of lymphedema conducted thereafter.

In 2010, findings from the PAL trial drew international attention (25). PAL was a randomized, controlled trial that involved 154 women treated for breast cancer between 1-5 years prior, and evaluated the effect of a 12month resistance exercise intervention.

Findings showed reduced lymphedema rates in the exercise group compared with the control group (11\% versus $17 \%$, respectively). While the difference in lymphedema cases between the two groups was clinically relevant, the findings were not supported statistically. In 2012, findings from the first exercise and lymphedema effectiveness trial (the Exercise for Health [EfH] trial) were published (26). EfH evaluated the effect of a pragmatic, 8month exercise intervention, commencing 6weeks post-surgery for breast cancer. The study involved two exercise groups and a control group, with those in the exercise groups participating in mixed-mode exercise (aerobic and resistance exercise) of moderate or higher intensity, delivered via face-to-face (exercise group 1) or telephone (exercise group 2) in 16 sessions over 8 months. Of note, the majority of the intervention was unsupervised even for those in the face-to-face exercise group. Findings showed no difference in lymphedema incidence by 12 -months post-diagnosis between the two exercise groups (11\%) and although again not supported statistically, incidence was lower in the exercise groups compared with the control group (16\%). These effectiveness findings suggest that even when exercise is conducted in unsupervised settings, exercise (including exercise at moderate or higher intensity) is safe for those at risk of lymphedema. However, the caveat to these findings was that all studies involved educating and guiding patients about appropriate starting exercise loads and intensities, and progressive overload.

In 2003 and 2005, the first results evaluating the acute $(n=31)(27)$ and chronic $(n=14)(28)$ responses to exercise in those with established lymphedema (specifically, unilateral breast cancer-related lymphedema) were published. These early findings suggested that exercise, including resistance training and exercise involving repetitive arm movement, were safe for those with lymphedema. Since the publication of these novel findings, there has been a rise in the number of studies evaluating the acute or chronic response to exercise for those with lymphedema, and together, the findings from these studies were the subject of a systematic review and metaanalysis published in 2016. The goal of the systematic review was to evaluate the effects of exercise on lymphedema and associated symptoms, as well as to determine the need to wear compression during exercise (29). Twelve studies that evaluated the acute effect of exercise (that is, a single bout/session of exercise) and 13 studies that evaluated the chronic effect of exercise (i.e., 4 weeks or longer of regular exercise training) on lymphedema-related outcomes were included. All except one of the 25 studies included involved women with unilateral upper-limb lymphedema; the remaining study involved men and women with lower-limb lymphedema. Exercise prescribed and evaluated was of moderate- to high-intensity, and although the majority of interventions were conducted under supervised conditions, results from homebased, unsupervised exercise also 
contributed to the review (30-32).

Approximately half of the studies evaluated resistance-based exercise alone or in combination with aerobic-based exercise. The remaining $50 \%$ evaluated a wide range of activities including walking, pole walking, floor-based aerobics, water-based exercise, yoga and tai chi, with theoretical underpinning typically behind the choice of exercise under investigation. For example, water-based exercise is considered particularly useful for lower-limb lymphedema as the pressure from the water may increase the effect of the exercise on lymph flow and counteract effects from gravity $(33,34)$. Pole walking is considered useful, particularly for upper-limb lymphedema, as it requires upper-limb muscular contractions related to holding and using the pole, while also stimulating the cardiovascular system (31). The key findings from the review were:

(i) Exercise was deemed safe for those with established lymphedema. However, there was no evidence to support that exercise reduced lymphedema. That is, neither an acute exercise bout nor participation in regular exercise of 4 weeks or longer exacerbated or reduced existing lymphedema. It was however suggested that participation in exercise may prevent lymphedema progression. These findings were consistent, irrespective of exercise mode.

(ii) There is insufficient evidence to support or refute current clinical recommendations to wear compression garments during exercise. That is, there were too few studies available, and of low scientific quality, that evaluated the effect of compression during exercise.

These findings led the authors to conclude:

(i) While much research attention has been given to resistance exercise in the context of lymphedema prevention and management, other modes of exercise seem as beneficial to lymphedema outcomes. Therefore, it is important to consider all exercise goals (that may or may not be related to lymphedema) that a patient may have and to identify optimal exercise prescription (including mode) in meeting these goals. It is also important to consider patients' exercise preferences, as this will undoubtedly influence compliance and adherence to any exercise program.

(ii) Given the well-established benefits of exercise post-cancer including improvements in function and quality of life, and potential survival benefits, those with lymphedema should follow physical activity and exercise recommendations promoted to all cancer patients. However, individualized starting loads and slow rate of progression (that is, progressively increasing duration, load or intensity in small increments) is advised.

(iii) Garment wear during exercise should also be considered on an individual basis, with the first rule being to wear compression during exercise unless exercising in the water. However, if compression prevents a patient from exercising because compression is deemed uncomfortable or too hot, it is better to refrain compression than to refrain from exercise.

Overall, it was concluded that exercise may prevent lymphedema, with no evidence suggesting exercise causes lymphedema. For those with lymphedema, exercise may not make it better, but it may prevent progression and there is no evidence that it makes lymphedema worse. So good news, but not fantastic news, and likely less than exercise scientists expected to observe. It is possible that the benefits of exercise on lymphedema are currently being limited by the characteristics of the exercise programs that have been evaluated to date, such as the intensity of the exercise or the frequency of the exercise sessions. For example, during resistance exercise, heavier loads are more effective at eliciting strength gains and physiological adaptations, compared with lighter loads, and at least in the lymphedema prevention setting, the majority of exercise interventions evaluated have been at moderate rather than high intensity. Consequently, Bloomquist and colleagues published findings from their randomized, cross-over equivalence trial, which evaluated the acute lymphatic 
response to a low- versus high-load resistance training session in women at risk of lymphedema following breast cancer. Findings showed a similar response irrespective of the exercise load, and that a dose of high load resistance exercise was safe for those at risk of lymphedema (35). We now await the findings from a randomized, exercise trial that explores the longer-term lymphatic response of high load resistance exercise for women at risk of lymphedema. Another factor potentially limiting positive exercise response is the frequency or the combination of intensity and frequency of exercise. Most exercise programs tested to date have involved low- or moderateintensity exercise, 1-3 times per week. The provision of a higher frequency stimulus (e.g., $>3$ times per week) may be required to instigate measurable benefits to the lymphatic system. Preliminary findings from a polewalking study that encouraged 3-5 sessions per week support this hypothesis (31).

Despite a mounting evidence base in support of exercise being safe for those at-risk of or with lymphedema, findings need to be considered in light of their limitations. First, there remains a need for studies with larger sample size, of higher scientific quality, and involving those with lymphedema beyond unilateral upper-limb lymphedema (including those with lower-limb lymphedema, secondary lymphedema other than cancer-related lymphedema, and primary lymphedema). Second, of the lymphedema samples studied to date, they are likely biased towards including those with more stable but persistent lymphedema (potentially difficult to modify via exercise) and/or mild lymphedema (restricts capacity to see benefit). Improvements in understanding the role of exercise would occur through targeting those with regular lymphedema exacerbations, but also through a more comprehensive description of lymphedema characteristics of study samples. Further, the vast majority of exercise intervention trials evaluating lymphedema outcomes have been undertaken in supervised settings and with progressive increases in load, intensity, and duration of exercise sessions over time. Lymphedema is a chronic disease that requires patient self-management. As such, there is a clear need for greater understanding of the safety and effect of exercise on lymphedema when undertaken in unsupervised settings.

As our understanding of structure and function of the lymphatic vessels, organs, tissues, and lymph improves, as well as the interrelationship between the lymphatic system and other physiological systems, so too will our understanding of the mechanisms by which exercise could benefit the prevention and treatment of lymphedema. Nonetheless, as research evolves, we can learn from the past and be guided by what's currently known while considering the unknowns. There is no doubt that lymphedema prevention and management guidelines endorsed today, generally encourage participation in physical activity. However, there remains scope for improvements. Guidelines should be used to educate about the positive relationship between participating in physical activity, including planned exercise, and overall health, function, survival, as well as what is known about exercise and lymphedema. Further, a change in the tone and language of guidelines is recommended - patients should first be advised on what they can and should do, rather than what they can't do. Any avoidance advice should be around avoiding sedentary behavior. Subtle changes made in this way could lead to even small, but significant positive changes in the mindsets of patients at risk or with lymphedema, including reduced fear associated with lymphedema.

In summary, evidence to date suggests that those at risk - or with lymphedema should look for opportunities to be physically active as often as possible and to participate in planned exercise regularly (that is, more often than not). Planned exercise should include aerobic- and resistance-exercise of any type, undertaken at moderate- or higher-intensity (that is, exercise that makes you huff and puff). It is appropriate to start with exercise 
sessions of short duration and lower intensities and to progress in small increments (by slowly increasing duration and/or intensity). Progression should be guided by lymphedema measurements and symptoms (subjectively or objectively identified) with worsening indicative of the need to follow-up with a lymphedema therapist.

\section{CONFLICT OF INTEREST AND DISCLOSURE}

The authors declare no competing financial interests exist.

\section{REFERENCES}

1. The Diagnosis and Treatment of Peripheral Lymphedema: 2020 Consensus Document of the International Society of Lymphology. Lymphology 53 (2020), 3-19.

2. Schulze, H, M Nacke, C Gutenbrunner, et al: Worldwide assessment of healthcare personnel dealing with lymphoedema. Health Econ. Rev. 8 (2018), 10.

3. DiSipio, T, S Rye, B Newman, et al: Incidence of unilateral arm lymphoedema after breast cancer: A systematic review and meta-analysis. Lancet Oncol. 14 (2013), 500-515.

4. Johansson, K, E Branje: Arm lymphoedema in a cohort of breast cancer survivors 10 years after diagnosis. Acta Oncol. 49(2) (2010), 166-173.

5. Hayes, SC, M Janda, LC Ward, et al: Lymphedema following gynecological cancer: Results from a prospective, longitudinal cohort study on prevalence, incidence and risk factors. Gynecol. Oncol. 146 (2017), 623-629.

6. Johansson, K, H Holmström, I Nilsson, et al: Breast cancer patients' experiences of lymphoedema. Scand. J. Caring Sci. 17 (2003), 35-42.

7. Boyages, J, Y Xu, S Kalfa, et al: Financial cost of lymphedema borne by women with breast cancer. Psychooncology 26 (2017), 849-855.

8. Carter, N, D Bryant-Lukosius, A DiCenso, et al: The use of triangulation in qualitative research. Oncol. Nurs. Forum 41 (2014), 545-547.

9. Kurz, I: Einfuhrung in die Manuelle Lymphdrainage nach Dr. Vodder. 3. Auflage. Heidelberg Karl F. Haug Verlag.
1984.

10. Larsson, IL, C Jönsson, AC Olsson, et al: Women's experience of physical activity following breast cancer treatment. Scand. J. Caring Sci. 22 (2008), 422-429.

11. Friedenreich, CM, CR Stone, WY Cheung, et al: Physical activity and mortality in cancer survivors: A systematic review and metaanalysis. JNCI Cancer Spectr. 4 (2019), pkz080.

12. Greenlee, H, JM Unger, M LeBlanc, et al: Association between body mass index and cancer survival in a pooled analysis of 22 clinical trials. Cancer Epidemiol. Biomarkers Prev. 26 (2017), 21-29.

13. Chan, DSM, AR Vieira, D Aune, et al: Body mass index and survival in women with breast cancer-systematic literature review and metaanalysis of 82 follow-up studies. Ann. Oncol. 25 (2014), 1901-1914.

14. Volaklis, KA, M Halle, C Meisinger: Muscular strength as a strong predictor of mortality: A narrative review. Eur. J. Intern. Med. 26 (2015), 303-310.

15. Schmid, D, MF Leitzmann: Cardiorespiratory fitness as predictor of cancer mortality: A systematic review and meta-analysis. Ann. Oncol. 26 (2015), 272-278.

16. Hayes, SC, RU Newton, RR Spence, et al: The Exercise and Sports Science Australia position statement: Exercise medicine in cancer management. J. Sci. Med. Sport 22 (2019), 1175-1199.

17. Campbell, KL, KM Winters-Stone, J Wiskemann, et al: Exercise Guidelines for Cancer Survivors: Consensus Statement from International Multidisciplinary Roundtable. Med. Sci. Sports Exerc. 51 (2019), 2375-2390.

18. Segal, R, C Zwaal, E Green, et al: Exercise for people with cancer: A clinical practice guideline. Curr. Oncol. 24 (2017), 40-46. Ivens,

19. D, AL Hoe, TJ Podd, et al: Assessment of morbidity from complete axillary dissection. Br. J. Cancer 66 (1992), 136-138.

20. Maunsell, E, J Brisson, L Deschênes: Arm problems and psychological distress after surgery for breast cancer. Can. J. Surg. 36 (1993), 315-320.

21. Johansson, K, C Ingvar, M Albertsson, et al: Arm lymphoedema, shoulder mobility and muscle strength after breast cancer treatment? A prospective 2-year study. Adv. Physiother. 3(2) (2001), 55-66.

22 McKenzie, DC: Abreast in a boat--a race against breast cancer. CMAJ 159 (1998), 376-378.

23. Harris, SR, SL Niesen-Vertommen: Challenging the myth of exercise-induced lymphedema following breast cancer: A series of case reports. J. Surg. Oncol. 74 (2000), 95-99. 
24. Box, RC, HM Reul-Hirche, JE BullockSaxton, et al: Physiotherapy after breast cancer surgery: Results of a randomised controlled study to minimise lymphoedema. Breast Cancer Res. Treat. 75 (2002), 51-64.

25. Schmitz, KH, RL Ahmed, AB Troxel, et al: Weight lifting for women at risk for breast cancer-related lymphedema: A randomized trial. JAMA 304 (2010), 2699-2705.

26. Hayes, SC, S Rye, T Disipio, et al: Exercise for health: A randomized, controlled trial evaluating the impact of a pragmatic, translational exercise intervention on the quality of life, function and treatmentrelated side effects following breast cancer. Breast Cancer Res. Treat. 137 (2013), 175-186.

27. Johansson, K, K Tibe, A Weibull, et al: Low intensity resistance exercise for breast cancer patients with arm lymphedema with or without compression sleeve. Lymphology 38 (2005), 167-180.

28. McKenzie, DC, AL Kalda: Effect of upper extremity exercise on secondary lymphedema in breast cancer patients: A pilot study. J. Clin. Oncol. 21 (2003), 463-466.

29. Singh, B, T Disipio, J Peake, et al: Systematic review and meta-analysis of the effects of exercise for those with cancerrelated lymphedema. Arch. Phys. Med. Rehabil. 97 (2016), 302-315.e13.

30. Gautam, AP, AG Maiya, MS Vidyasagar: Effect of home-based exercise program on lymphedema and quality of life in female post mastectomy patients: Pre-post intervention study. J. Rehabil. Res. Dev. 48 (2011), 1261-1268.
31. Jönsson, C, K Johansson: The effects of pole walking on arm lymphedema and cardiovascular fitness in women treated for breast cancer: A pilot and feasibility study. Physiother. Theory Pract. 30 (2014), 236-242.

32. Johansson, K, P Klernäs, A Weibull, et al: A home-based weight lifting program for patients with arm lymphedema following breast cancer treatment: a pilot and feasibility study. Lymphology 47 (2014), 51-64.

33. Johansson, K, S Hayes, RM Speck, et al: Water-based exercise for patients with chronic arm lymphedema: A randomized controlled pilot trial. Am. J. Phys. Med. Rehabil. 92 (2013), 312-319.

34. Tidhar, D, M Katz-Leurer: Aqua lymphatic therapy in women who suffer from breast cancer treatment-related lymphedema: A randomized controlled study. Support. Care Cancer. 18 (2010), 383-392.

35. Bloomquist, K, P Oturai, ML Steele, et al: Heavy-load lifting: Acute response in breast cancer survivors at risk for lymphedema. Med Sci Sports Exerc 50 (2018), 187-195.

\author{
Sandi Hayes, PhD, Professor \\ Menzies Health Institute Queensland \\ G40 Griffith Health Centre Level 8.86 \\ Gold Coast Campus Griffith University \\ Queensland, Australia 4222 \\ Phone: +617 56788811 \\ E-Mail: sandi.hayes@griffith.edu.au
}

